\title{
Time dependent delta-prime interactions in dimension one
}

\author{
C. Cacciapuoti $^{1}$, A. Mantile ${ }^{2}$, A. Posilicano ${ }^{1}$ \\ ${ }^{1}$ DiSAT, Sezione di Matematica, Università dell'Insubria, \\ via Valleggio 11, 22100 Como, Italy \\ ${ }^{2}$ Laboratoire de Mathématiques, Université de Reims - FR3399 CNRS, \\ Moulin de la Housse BP 1039, 51687 Reims, France \\ claudio.cacciapuoti@uninsubria.it, andrea.mantile@univ-reims.fr, \\ andrea.posilicano@uninsubria.it
}

PACS 02.30.Jr, 03.65.Db, 02.30.Rz

DOI 10.17586/2220-8054-2016-7-2-303-314

\begin{abstract}
We solve the Cauchy problem for the Schrödinger equation corresponding to the family of Hamiltonians $H_{\gamma(t)}$ in $L^{2}(\mathbb{R})$ which describes a $\delta^{\prime}$-interaction with time-dependent strength $1 / \gamma(t)$. We prove that the strong solution of such a Cauchy problem exists whenever the map $t \mapsto \gamma(t)$ belongs to the fractional Sobolev space $H^{3 / 4}(\mathbb{R})$, thus weakening the hypotheses which would be required by the known general abstract results. The solution is expressed in terms of the free evolution and the solution of a Volterra integral equation.
\end{abstract}

Keywords: time dependent point interactions, delta-prime interaction, non-autonomous Hamiltonians.

Received: 10 February 2016

\section{Introduction}

In this paper, we address the generation problem for the family of time-dependent Hamiltonians $H_{\gamma(t)}$, where $H_{\gamma(t)}$, for any fixed real $t$, denotes the self-adjoint operator in $L^{2}(\mathbb{R})$ describing a $\delta^{\prime}$-interaction of strength $1 / \gamma(t)$ (see [1,2], [3, Chapter I.4] and references therein).

Most of the literature on time dependent point interactions focuses on perturbations of the free dynamics of the form of a Dirac's delta time dependent potential. In three dimensions time dependent $\delta$-interactions were studied in [4,5] and in [6] in relation with the problem of ionization under periodic perturbations, see also [7]. In two dimensions, very recently, the problem of the well-posedness was studied in [8]. In one dimension, this kind of nonautonomouss Hamiltonians was analyzed in [9], see also [10].

It is well known that in one dimension, the family of point perturbations of the Laplacian is richer than in two and three dimensions, and includes $\delta$ and $\delta^{\prime}$ perturbations, as well as their combinations. In this paper, we focus attention on the topical case of a time dependent $\delta^{\prime}$ interaction.

We remark that time-dependent $\delta$-interactions have a non-linear counterpart, see, e.g., [11-13] in three dimensions, and [14,15]. More recently, a systematic study of the blow-up in the one dimensional case was started in [16]. In one dimension, in particular, such models find applications to the propagation of optical waves in Kerr media, or one-dimensional many body systems, see, e.g., [17-20] and references therein. The problem of the derivation of non-linear $\delta$-interactions from scaled regular dynamics was recently studied in one- and threedimensions [21-23].

Several results discussed in the present paper set the groundworks for defining the nonlinear point interactions of $\delta^{\prime}$-type and for the study of the problem of their derivation from scaled regular dynamics. 
We recall that the definition of $H_{\gamma}$ is given by the theory of self-adjoint extensions of the symmetric operator:

$$
H^{\circ}=-\Delta \equiv-\frac{d^{2}}{d x^{2}}, \quad D\left(H^{\circ}\right)=C_{0}^{\infty}(\mathbb{R} \backslash\{0\}),
$$

and, for any real $\gamma$, reads as follows:

$$
\begin{gathered}
H_{\gamma} \psi(x)=-\frac{d^{2} \psi}{d x^{2}}(x), \quad x \neq 0, \\
D\left(H_{\gamma}\right)=\left\{\psi \in L^{2}(\mathbb{R}): \psi=\phi+q \eta, \phi \in X^{2}, q \in \mathbb{C}, \phi^{\prime}(0)=\gamma q\right\},
\end{gathered}
$$

where $\eta(x):=\frac{1}{2} \operatorname{sgn}(x)$ and for any $\nu \geq 0$ we defined $X^{\nu}$ as the space of tempered distributions with Fourier transform in $L^{2}\left(\mathbb{R},|k|^{2 \nu} d k\right)$.

We remark that if $f \in X^{\nu}$, then its Fourier transform might be a distribution as well. Moreover, for $\nu=m+\sigma$, with $m$ integer and $1 / 2<\sigma \leq 1$, if $f \in X^{\nu}$ then $f \in C^{m}(\mathbb{R})$, see Prop. 2.1 below. Hence, $\phi$ in $D\left(H_{\gamma}\right)$ is a $C^{1}(\mathbb{R})$ function and $\phi^{\prime}(0)$ in the boundary condition is well defined.

The action of the operator $H_{\gamma}$ can be understood also by exploiting the decomposition $\psi=\phi+q \eta$ : this leads to

$$
H_{\gamma} \psi(x)=-\phi^{\prime \prime}(x), \quad x \in \mathbb{R} .
$$

When $\gamma(t)$ is assigned as a real valued function of time, the domain $D\left(H_{\gamma(t)}\right)$ changes in time with the boundary condition $\phi^{\prime}(0)=\gamma(t) q$. In contrast, the quadratic form corresponding to $H_{\gamma}$ is given by

$$
\begin{gathered}
Q_{\gamma}(\psi)=\left\|\phi^{\prime}\right\|^{2}+\gamma|q|^{2} \\
D\left(Q_{\gamma}\right)=\left\{\psi \in L^{2}(\mathbb{R}): \psi=\phi+q \eta, \phi \in X^{1}, q \in \mathbb{C}\right\},
\end{gathered}
$$

and so $Q_{\gamma(t)}$ has a time-independent domain. Thus, by the abstract results in [24] and [10], assuming that the map $t \mapsto \gamma(t)$ is differentiable, there exists an unitary propagator $U_{t, s}$ in $L^{2}(\mathbb{R})$, continuously mapping $D\left(H_{\gamma(s)}\right)$ onto $D\left(H_{\gamma(t)}\right)$, such that $\psi(t):=U_{t, 0} \psi_{0}$ is the (strong) solution of the Cauchy problem:

$$
\left\{\begin{array}{l}
i \frac{d}{d t} \psi(t)=H_{\gamma(t)} \psi(t) \\
\psi(0)=\psi_{0} \in D\left(H_{\gamma(0)}\right) .
\end{array}\right.
$$

However, as the case of time-dependent self-adjoint extensions $H_{\alpha(t)}$ (corresponding to a $\delta$-interaction) studied in [9] suggests, the quite explicit knowledge of the action and operator domain of $H_{\gamma}$ should allow one to solve the Cauchy problem (4) under weaker regularity conditions on $\gamma(t)$. Indeed, as we show in this paper, this is the case and problem (4) has a strong, unique solution whenever the map $t \mapsto \gamma(t)$ is in the fractional Sobolev space $H^{3 / 4}(\mathbb{R})$, a condition weaker than the differentiability hypotheses required in [24] and [10]. Such a $H^{3 / 4}$ hypothesis is the same required in the paper [9] in order to guarantee that the Cauchy problem for the family $H_{\alpha(t)}$ has a strong solution. However, in contrast to [9], here we make use neither of sophisticated analytic tools (paraproducts) nor of abstract generation theorems (as the ones provided in [24] and [25]); instead, following the same strategy as in the paper [26], we apply a more direct approach which exploits definitions (1) and (2), providing a relatively explicit expression for the solution of (4) with initial datum $\psi_{0}=\phi_{0}+q_{0} \eta$ in $D\left(H_{\gamma(0)}\right)$ : 


$$
\psi(t)=\phi(t)+q(t) \eta
$$

with

$$
\phi(t)=e^{i t \Delta} \phi_{0}-\int_{0}^{t} d s \dot{q}(s) e^{i(t-s) \Delta} \eta,
$$

where $t \mapsto q(t)$ solves the Volterra-type integral equation

$$
q(t)=f_{0}(t)-\sqrt{\frac{4 i}{\pi}} \int_{0}^{t} d s \frac{\gamma(s) q(s)}{\sqrt{t-s}}
$$

and the source term $f_{0}$ is defined as:

$$
f_{0}(t):=q_{0}+\sqrt{\frac{4 i}{\pi}} \int_{0}^{t} d s \frac{\left(e^{i s \Delta} \phi_{0}\right)^{\prime}(0)}{\sqrt{t-s}} .
$$

We shall prove the following well-posedness result:

Theorem 1. Let $T>0, \gamma \in H^{3 / 4}(0, T)$, and set $\gamma_{0}=\gamma(0)$. Let $\psi_{0}=\phi_{0}+q_{0} \eta \in D\left(H_{\gamma_{0}}\right)$. Then, for any $t \in[0, T]$, there exists a unique strong solution for the Cauchy problem (4) given by $\psi(t)=\phi(t)+q(t) \eta$ as in Eqs. (6) - (8). Moreover, the map $t \mapsto H_{\gamma(t)} \psi(t)$ belongs to $C\left([0, T], L^{2}(\mathbb{R})\right)$.

We briefly discuss the heuristic derivation of the solution. The solution of the Schrödinger equation with $H_{\gamma}$ as Hamiltonian satisfies the distributional equation:

$$
i \frac{\partial}{\partial t} \psi(t)=-\psi^{\prime \prime}(t)+q(t) \delta_{0}^{\prime}
$$

where $\delta_{0}^{\prime}$ is the first derivative of the Dirac delta-distribution. Let us assume, in the first part of this discussion, that the source term $q(t)$ is an assigned function. Since $\eta^{\prime \prime}=\delta_{0}^{\prime}$, it is natural to seek for solutions of the form (5). Setting $\psi(t)=\phi(t)+q(t) \eta$ in Eq. (9) gives the following equation for $\phi(t)$ :

$$
i \frac{\partial}{\partial t} \phi(t)=-\phi^{\prime \prime}(t)-i \dot{q}(t) \eta
$$

Eq. (6) follows directly from the Duhamel's formula. Indeed by integration by parts, see Section 2.3 (in particular Eqs. (15) and (17)), one obtains the following equation for $\psi(t)$ :

$$
\psi(t)=e^{i t \Delta} \psi_{0}-i \int_{0}^{t} d s q(s) e^{i(t-s) \Delta} \delta_{0}^{\prime} .
$$

This can be understood as Duhamel's formula applied to Eq. (9).

The equation for $q(t)$ is obtained by imposing the boundary condition $\phi^{\prime}(0)=\gamma q$, using Eq. (6) to compute the 1.h.s. in the boundary condition. We postpone the details of the calculation to Section 2.3. Here we note that the boundary condition turns the flow associated to Eq. (9) into a unitary flow. In fact, one can show that:

$$
\frac{d}{d t}\|\psi(t)\|^{2}=2 \operatorname{Im} \bar{q}(t) \phi^{\prime}(0, t)
$$


Hence, if the boundary condition is satisfied, one has $\frac{d}{d t}\|\psi(t)\|=0$.

We remark that a function $\psi \in D\left(H_{\gamma}\right)$ can be written as the sum of a regular and singular part with both functions in $L^{2}$ by introducing a regularization parameter $\lambda$. More precisely, define:

$$
G_{\lambda}(x):=-\frac{e^{-\sqrt{\lambda}|x|}}{2 \sqrt{\lambda}} \quad \lambda>0 .
$$

The function $G_{\lambda}$ is the solution of the distributional equation $G_{\lambda}^{\prime \prime}=\delta_{0}+\lambda G_{\lambda}$. The domain $D\left(H_{\gamma}\right)$ can be rewritten as

$$
D\left(H_{\gamma}\right)=\left\{\psi \in L^{2}(\mathbb{R}): \psi=\phi_{\lambda}+q G_{\lambda}^{\prime}, \phi_{\lambda} \in H^{2}(\mathbb{R}), q \in \mathbb{C}, \phi_{\lambda}^{\prime}(0)=\left(\gamma+\frac{\sqrt{\lambda}}{2}\right) q\right\},
$$

and the action of $H_{\gamma}$ can be understood by the identity:

$$
\left(H_{\gamma}+\lambda\right) \psi(x)=-\phi_{\lambda}^{\prime \prime}(x)+\lambda \phi_{\lambda}(x), \quad x \in \mathbb{R},
$$

see, e.g., [3]. Eq. (3) is obtained by taking $\lambda \rightarrow 0$.

We note that the charge equation (7) does not depend on $\lambda$, it is easy to see that:

$$
f_{0}(t)=\sqrt{\frac{4 i}{\pi}} \int_{0}^{t} d s \frac{\left(e^{i s \Delta} \psi_{0}\right)^{\prime}(0)}{\sqrt{t-s}},
$$

see Eqs. (18) and (19) below. The equation for the regular part $\phi_{\lambda}$, instead, does involve the regularization parameter, precisely

$$
\phi_{\lambda}(t)=e^{i t \Delta} \phi_{\lambda, 0}-\int_{0}^{t} d s(\dot{q}(s)+\lambda q(s)) e^{i(t-s) \Delta} G_{\lambda}^{\prime} .
$$

We note that, even if the regularization would avoid few issues with convolutions and Fourier transforms, which must otherwise be interpreted in distributional sense, it makes formulae more involved and introduces an unnecessary parameter. For those reasons, we decided to avoid it.

The paper consists of one additional section in which we prove Theorem 1 .

\section{Proof of Theorem 1}

\subsection{Notation and preliminaries} line to line.

In what follows, $C$ denotes a generic positive constant whose value may change from

We denote by $\hat{\psi}$ the spatial Fourier transform of $\psi$ :

$$
\hat{\psi}(k)=\int_{\mathbb{R}} d x e^{-i k x} \psi(x) .
$$

The time-Fourier transform of $f$ is denoted by $\mathcal{F} f$ and defined as: 


$$
\mathcal{F} f(\omega)=\int_{\mathbb{R}} d t e^{-i \omega t} f(t) .
$$

With these definitions, the Fourier transform of the convolution is:

$$
(\widehat{\psi * \phi})(k)=\hat{\psi}(k) \hat{\phi}(k),
$$

and similarly for the time-Fourier transform.

In the following, we denote by $U(t)$ the free unitary group $e^{i \Delta t}$, we recall that its explicit expression is given by:

$$
U(t) \psi(x)=\int_{\mathbb{R}} d y \frac{e^{\frac{i(x-y)^{2}}{4 t}}}{\sqrt{4 \pi i t}} \psi(y),
$$

which in Fourier transform reads:

$$
\widehat{U(t) \psi}(k)=e^{-i k^{2} t} \hat{\psi}(k) .
$$

Proposition 2.1. For $\nu=m+\sigma$, with $m$ integer and $1 / 2<\sigma \leq 1$, it results $X^{\nu} \subset C^{m}(\mathbb{R})$.

Proof. In Fourier transform:

$$
f^{(m)}(x)-f^{(m)}(y)=\frac{1}{2 \pi} \int_{\mathbb{R}} d k(i k)^{m}\left(e^{i k x}-e^{i k y}\right) \hat{f}(k) .
$$

We note that:

Moreover:

$$
\begin{aligned}
\left|\int_{k \mid<1} d k(i k)^{m}\left(e^{i k x}-e^{i k y}\right) \hat{f}(k)\right| & \leq C|x-y|^{\sigma} \int_{|k|<1} d k|k|^{m+\sigma}|\hat{f}(k)| \\
& \leq C|x-y|^{\sigma}\|\hat{f}\|_{L^{2}\left(\mathbb{R},|k|^{2 \nu} d k\right)} .
\end{aligned}
$$

$$
\begin{aligned}
\int_{|k|>1} d k(i k)^{m}\left(e^{i k x}-e^{i k y}\right) \hat{f}(k) \mid & \leq C \int_{|k|>1} d k|k|^{m}|\hat{f}(k)| \\
& \leq C\left(\int_{|k|>1} \frac{d k}{|k|^{2 \sigma}}\right)^{\frac{1}{2}}\|\hat{f}\|_{L^{2}\left(\mathbb{R},|k|^{2 \nu} d k\right)} .
\end{aligned}
$$

Then, the continuity of $f^{(m)}$ follows from the bounds (10) and (11), and the dominated convergence theorem.

We will make use of fractional Sobolev spaces; for this reason we recall few definitions. For any $-\infty \leq a<b \leq+\infty$ and $\nu \in(0,1)$, we set:

$$
[f]_{H^{\nu}(a, b)}:=\left(\int_{[a, b]^{2}} d s d s^{\prime} \frac{\left|f(s)-f\left(s^{\prime}\right)\right|^{2}}{\left|s-s^{\prime}\right|^{1+2 \nu}}\right)^{1 / 2}
$$


which is sometimes referred to as Gagliardo (semi)norm of $f$. The space $H^{\nu}(a, b)$, for $-\infty \leq$ $a<b \leq+\infty$ and $\nu \in(0,1)$, is the space of functions for which the norm

$$
\|f\|_{H^{\nu}(a, b)}=\|f\|_{L^{2}(a, b)}+[f]_{H^{\nu}(a, b)}
$$

is finite. To define the space $H^{\nu}(a, b)$ for $\nu>1$ not integer, one sets $\nu=m+\sigma$, where $m$ is an integer and $\sigma \in(0,1)$. Then $H^{\nu}(a, b)$ is the space of functions such that $f \in H^{m}(a, b)$ and $f^{(m)} \in H^{\sigma}(a, b)$.

Remark 2.2. Note that, for $\nu \in(0,1)$ there exists a constant $C_{\nu}$ such that:

$$
[f]_{H^{\nu}(\mathbb{R})}=C_{\nu}\|\mathcal{F} f\|_{L^{2}\left(\mathbb{R},|\omega|^{2 \nu} d \omega\right)},
$$

for any $f \in X^{\nu}$, this is a direct consequence of Plancherel's theorem (see [27], Proposition 1.37). This identity, together with Prop. 2.1 implies that, for all $\nu>1 / 2$, and $a$ and $b$ finite, if $f \in X^{\nu}$ then $f \in H^{\nu}(a, b)$, and, consequently, it belongs to $H^{\mu}(a, b)$ for all $0 \leq \mu \leq \nu$. Also, if $f \in L^{2}(a, b)$ and $f \in X^{\nu}$, then $f \in H^{\nu}(a, b)$, and, consequently, in $H^{\mu}(a, b)$ for all $0 \leq \mu \leq \nu$.

We recall that, for $-\infty \leq a<b \leq+\infty$, the space $L^{2}(a, b)$ can be identified with $H^{0}(a, b)$, and $L^{2}(\mathbb{R})$ can be identified with $X^{0}$.

For the norms, we shall use the notation $\|\cdot\|=\|\cdot\|_{L^{2}(\mathbb{R})}$.

We denote by $I$ the operator:

$$
I f(t)=\frac{1}{\sqrt{\pi}} \int_{0}^{t} d s \frac{f(s)}{\sqrt{t-s}} .
$$
operator $I$.

We shall use the following results which establish the regularization properties of the

Lemma 2.3. Let $\nu \geq 0$ and $T>0$. Assume that $f \in X^{\nu}$ and has support in $[0, T]$, then If $\in X^{\nu+1 / 2}$.

Proof. The integral kernel:

$$
A(t)=\frac{1}{\sqrt{\pi}} \frac{\Theta(t)}{\sqrt{t}}
$$

where $\Theta$ is the Heaviside function, is a tempered distribution and

$$
\mathcal{F} A(\omega)=\frac{1}{\sqrt{|\omega|}}\left(\frac{\sqrt{i}}{2} \Theta(\omega)+\frac{1}{\sqrt{2}}(\Theta(-\omega)+i \Theta(\omega))\right) .
$$

Let $f \in X^{\nu}$. The convolution of $A$ and $f$, If $=A * f$, is a tempered distributions and $\mathcal{F} I f=\mathcal{F} A \mathcal{F} f$, see, e.g., [28, Th. 14.25]. Then,

$$
\left\||\cdot|^{\nu+1 / 2} \mathcal{F} I f\right\| \leq C\left\||\cdot|^{\nu} \mathcal{F} f\right\|
$$

We recall the following technical lemma:

Lemma 2.4. Let $-\infty<a<b<\infty$ and let $f \in H^{\nu}(a, b)$ with $\nu \geq 0$. Define

$$
\tilde{f}(s)= \begin{cases}f(s) & \text { if } s \in[a, b] \\ 0 & \text { otherwise. }\end{cases}
$$

i) If $0 \leq \nu<1 / 2$, then $\tilde{f} \in H^{\nu}(\mathbb{R})$. 
ii) If $1 / 2<\nu<3 / 2$ and $f(a)=f(b)=0$, then $\tilde{f} \in H^{\nu}(\mathbb{R})$.

For the proof, see for example [29, Th. 11.4], see also [30, Th. III.3.2].

We shall also use the following:

Proposition 2.5. Let $\mu>1 / 2$ and $0 \leq \nu \leq \mu$. If $g \in H^{\mu}(a, b)$ and $f \in H^{\nu}(a, b)$ then $f g \in H^{\nu}(a, b)$.

For the proof we refer to [30].

\subsection{Well-posedness of the charge equation}

In this section, we study the well-posedness of the charge equation (7).

We start with the following lemma, which gives the regularity properties of the inhomogeneous term in Eq. (7):

Lemma 2.6. Let $\phi_{0} \in X^{2}$, then $\left(U(\cdot) \phi_{0}\right)^{\prime}(0) \in X^{3 / 4}$.

Proof. Since $\phi_{0}^{\prime} \in L^{2}(\mathbb{R})$, one has that the distributional identity:

$$
\left(U(t) \phi_{0}\right)^{\prime}(x)=\int_{\mathbb{R}} d y \frac{e^{\frac{i(x-y)^{2}}{4 t}}}{\sqrt{4 \pi i t}} \phi_{0}^{\prime}(y)
$$

shows that $\left(U(t) \phi_{0}\right)^{\prime} \in L^{2}(\mathbb{R})$. By using the Fourier transform, one has that:

$$
\left(U(t) \phi_{0}\right)^{\prime}(0)=\frac{1}{2 \pi} \int_{\mathbb{R}} d k e^{-i k^{2} t} \widehat{\phi}_{0}^{\prime}(k) .
$$

By splitting the integral in $d k$ for $k>0$ and $k<0$, and by using the change of variables $k=\sqrt{\omega}$ for $k>0$ and $k=-\sqrt{\omega}$ for $k<0$, it follows that:

$$
\left(U(t) \phi_{0}\right)^{\prime}(0)=\frac{i}{4 \pi} \int_{0}^{\infty} \frac{d \omega}{\sqrt{\omega}} e^{-i \omega t}\left(\widehat{\phi}_{0}^{\prime}(\sqrt{\omega})+\widehat{\phi}_{0}^{\prime}(-\sqrt{\omega})\right)
$$

Hence:

$$
\mathcal{F}\left(\left(U(\cdot) \phi_{0}\right)^{\prime}(0)\right)(\omega)=\frac{i}{2 \sqrt{\omega}} \Theta(-\omega)\left(\widehat{\phi}_{0}^{\prime}(\sqrt{-\omega})+\widehat{\phi}_{0}^{\prime}(-\sqrt{-\omega})\right)
$$

where $\Theta$ denotes the Heaviside function. To prove that $\mathcal{F}\left(\left(U(\cdot) \phi_{0}\right)^{\prime}(0)\right) \in L^{2}\left(\mathbb{R},|\omega|^{\frac{3}{2}} d \omega\right)$, it is enough to note that

$$
\left\||\cdot|^{\frac{3}{4}} \mathcal{F}\left(\left(U(\cdot) \phi_{0}\right)^{\prime}(0)\right)\right\| \leq C\left\||\cdot| \widehat{\phi_{0}^{\prime}}\right\|=C\left\||\cdot|^{2} \hat{\phi}_{0}\right\|
$$

where we used the change of variables $k^{2}=\omega$.

We are now ready to prove the main result of this section.

Lemma 2.7. Let $T>0, \gamma \in H^{3 / 4}(0, T)$, and set $\gamma_{0}=\gamma(0)$. Let $\psi_{0}=\phi_{0}+q_{0} \eta \in D\left(H_{\gamma_{0}}\right)$. Then, Eq. (7) admits a unique solution $q \in H^{5 / 4}(0, T)$.

Proof. We split the proof in two steps: first, we prove that there exists a unique solution $q \in L^{2}(0, T)$, then, by a bootstrap argument, we show that such solution belongs to $H^{5 / 4}(0, T)$.

We start by step 1 . We use several results from the monograph [31]. We set:

$$
k(t, s)=\sqrt{\frac{4 i}{\pi}} \frac{\gamma(s)}{\sqrt{t-s}}
$$


and rewrite the equation as:

$$
q(t)=f_{0}(t)-\int_{0}^{t} d s k(t, s) q(s) .
$$

This is a linear nonconvolution Volterra equation to which we can apply the results in [31, Ch. 9]. We start by noticing that for any finite interval $J \subset \mathbb{R}^{+}, k(t, s)$ is a Volterra kernel of type $L^{2}$, more precisely:

$$
\left\|| | k \left|\left\|_{L^{2}(J)}:=\sup _{\substack{\|h\|_{L^{2}(J)} \leq 1 \\\|g\|_{L^{2}(J)} \leq 1}} \int_{J} \int_{J} d s d t|h(t) k(t, s) g(s)| \leq C|J|^{1 / 2}\right\| \gamma \|_{L^{\infty}(J)} .\right.\right.
$$

Hence, the interval $[0, T]$ can be divided into finitely many subintervals $J_{i}$ such that $\||k|\|_{L^{2}\left(J_{i}\right)}<1$ on each $J_{i}$, and, as a consequence of Cor. 9.3.14 in [31], one has that $k$ has a resolvent of type $L^{2}$ on $[0, T]$. By applying Th. 9.3.6 of [31], we conclude that Eq. (13) has a unique solution in $L^{2}(0, T)$.

We can now proceed to the second step of the proof, which consists in showing that such a solution belongs to $H^{5 / 4}(0, T)$. By Lemma 2.6 and Rem. 2.2, one has $\left(U(\cdot) \phi_{0}\right)^{\prime}(0) \in H^{\nu}(0, T)$ for all $0 \leq \nu \leq 3 / 4$. We set:

$$
Q(t)=q(t)-q_{0} \quad \text { and } \quad F(t)=\sqrt{4 i}\left(\left(U(t) \phi_{0}\right)^{\prime}(0)-\gamma(t) q(t)\right) \quad t \in[0, T] .
$$

We denote by $\tilde{Q}$ the function obtained by prolonging $Q$ to zero outside $[0, T]$ and remark that the claim $\tilde{Q} \in X^{\nu}$ implies $Q \in H^{\mu}(0, T)$ for all $0 \leq \mu \leq \nu$, see Rem. 2.2, therefore $q \in H^{\mu}(0, T)$.

By the charge equation (7), the identity $Q=I F$ holds true for a.a. $t \in[0, T]$, here $I$ is the operator defined in (12). Since, by Prop. 2.5, $F \in L^{2}(0, T)$ we can define $\tilde{F} \in L^{2}(\mathbb{R})$ by extending it to zero. Then, by Lemma $2.3, \tilde{Q}=I \tilde{F} \in X^{1 / 2}$, hence, $Q \in H^{1 / 4}(0, T)$ and $q \in H^{1 / 4}(0, T)$.

We can repeat the argument. We start with the observation that now we know that $F \in H^{1 / 4}(0, T)$ and conclude that $q \in H^{3 / 4}(0, T)$. Here, we use Lemma 2.4-i) to claim that $\tilde{F} \in H^{1 / 4}(\mathbb{R})$ which in turn implies $\tilde{F} \in X^{1 / 4}$.

To conclude the proof, we must slightly adjust the argument above. So far, we have proved that $F \in H^{3 / 4}(0, T)$, moreover we know that $F(0)=0$, because the boundary condition $\phi_{0}^{\prime}(0)=\gamma_{0} q_{0}$ holds true by assumption. Define $F^{s}:[0,2 T] \rightarrow \mathbb{C}$ by reflection of $F$ about $t=T$. We have that $F^{s}(0)=F^{s}(2 T)=0$. We define $\tilde{F}^{s}: \mathbb{R} \rightarrow \mathbb{C}$ by extending $F^{s}$ to zero and use Lemma 2.4-ii) to claim that $\tilde{F}^{s} \in H^{3 / 4}(\mathbb{R})$, and, consequently, $\tilde{F} \in X^{3 / 4}$. Reapplying Lemma 2.3, we conclude that $q \in H^{5 / 4}(0, T)$.

\subsection{Proof of Theorem 1}

The function $\phi(t)$ defined by Eq. (6) exists and is unique for all $t \in[0, T]$. Next we prove that $\phi(t) \in X^{2}$. Let us rewrite Eq. (6) as:

$$
\phi(t)=U(t) \phi_{0}+\tilde{\phi}(t)
$$

where we set: 


$$
\tilde{\phi}(t)=-\int_{0}^{t} d s \dot{q}(s) U(t-s) \eta
$$

One has that $U(t) \phi_{0} \in X^{2}$, because $\left\|\widehat{U(t) \phi_{0}}\right\|_{L^{2}\left(\mathbb{R},|k|^{4} d k\right)}=\left\|\hat{\phi}_{0}\right\|_{L^{2}\left(\mathbb{R},|k|^{4} d k\right)}$.

We are left to prove that $\tilde{\phi} \in X^{2}$. We recall that the Fourier transform of $\eta$ is the distribution $-i \mathrm{PV} \frac{1}{k}$ (where PV stands for principal value). We have that:

$$
\begin{aligned}
\|\hat{\tilde{\phi}}(t)\|_{L^{2}\left(\mathbb{R},\left|k^{4}\right| d k\right)}^{2} & =\frac{1}{2 \pi} \int_{\mathbb{R}} d k k^{2}\left|\int_{0}^{t} d s e^{-i k^{2}(t-s)} \dot{q}(s)\right|^{2} \\
& =\frac{1}{2 \pi} \int_{0}^{\infty} d \omega \omega^{\frac{1}{2}}\left|\int_{0}^{t} d s e^{i \omega s} \dot{q}(s)\right|^{2} \leq C\|\dot{q}\|_{H^{1 / 4}(0, T)} .
\end{aligned}
$$

Here, the inequality follows from the same argument used in the proof of Prop. 3.3 in [23].

Next, we prove that $\psi(t)=\phi(t)+q(t) \eta \in L^{2}(\mathbb{R})$. Since $\phi(t) \in C^{1}(\mathbb{R})$, see Prop. 2.1, and $\eta$ is bounded, $\psi(t) \in L_{l o c}^{2}(\mathbb{R})$. Hence, it is enough to prove that $(1-\chi) \psi(t) \in L^{2}(\mathbb{R})$, where $\chi$ is the characteristic function of the interval $[-1,1]$. In the definition of $\phi(t)$, see Eq. (6), we use the identity:

$$
\int_{0}^{t} d s \dot{q}(s) U(t-s) \eta=q(t) \eta-q_{0} U(t) \eta-\int_{0}^{t} d s q(s) \frac{\partial}{\partial s} U(t-s) \eta
$$

which gives:

$$
\psi(t)=U(t) \psi_{0}+\int_{0}^{t} d s q(s) \frac{\partial}{\partial s} U(t-s) \eta
$$

Since $U(t) \psi_{0} \in L^{2}(\mathbb{R})$, we are left to prove that the second term at the r.h.s., times the function $(1-\chi)$, is also in $L^{2}(\mathbb{R})$. We note that:

From which, we get:

$$
\begin{aligned}
(U(t) \eta)(x) & =\int_{\mathbb{R}} d y \frac{e^{i \frac{(x-y)^{2}}{4 t}}}{\sqrt{4 \pi i t}} \eta(y) \\
& =\frac{1}{2} \frac{1}{\sqrt{4 \pi i t}}\left(\int_{-\infty}^{x} d y e^{i \frac{y^{2}}{4 t}}-\int_{x}^{\infty} d y e^{i \frac{y^{2}}{4 t}}\right) .
\end{aligned}
$$

$$
\frac{\partial}{\partial t}(U(t) \eta)(x)=-\frac{1}{2} \frac{1}{\sqrt{4 \pi i}} \frac{x}{t^{3 / 2}} e^{i \frac{x^{2}}{4 t}}=-\sqrt{\frac{i}{\pi}} \frac{\sqrt{t}}{x} \frac{d}{d t} e^{i \frac{x^{2}}{4 t}}
$$

We remark that the first equality can be understood in distributional sense as:

$$
\frac{\partial}{\partial t}(U(t) \eta)=i(U(t) \eta)^{\prime \prime}=i\left(U(t) \eta^{\prime \prime}\right)=i\left(U(t) \delta_{0}^{\prime}\right)
$$

from which, one deduces that Eq. (15) is equivalent to Eq. (5). 
This then gives:

$$
\begin{aligned}
& \int_{0}^{t} d s q(s) \frac{\partial}{\partial t}(U(t-s) \eta)(x)=\sqrt{\frac{i}{\pi} \frac{1}{x}} \int_{0}^{t} d s q(s) \sqrt{t-s} \frac{d}{d s} e^{i \frac{x^{2}}{4(t-s)}} \\
= & \sqrt{\frac{i}{\pi}} \frac{1}{x}\left(-q_{0} \sqrt{t} e^{i \frac{x^{2}}{4 t}}-\int_{0}^{t} d s \dot{q}(s) \sqrt{t-s} e^{i \frac{x^{2}}{4(t-s)}}+\frac{1}{2} \int_{0}^{t} d s \frac{q(s)}{\sqrt{t-s}} e^{i \frac{x^{2}}{4(t-s)}}\right) .
\end{aligned}
$$

We gained a factor $1 / x$ which gives the bound:

$$
\left\|(1-\chi) \int_{0}^{t} d s q(s) \frac{\partial}{\partial t} U(t-s) \eta\right\| \leq C\left(\|q\|_{L^{\infty}(0, T)}+\|\dot{q}\|_{L^{1}(0, T)}\right) \leq C \quad t \in[0, T] .
$$

Next, we prove that the boundary condition $\phi^{\prime}(0)=\gamma(t) q$ holds true for all $t \in[0, T]$. From Eq. (16), we obtain:

$$
(U(t) \eta)^{\prime}(0)=\frac{1}{\sqrt{4 \pi i t}}
$$

hence

$$
\phi^{\prime}(0, t)=\left(U(t) \phi_{0}\right)^{\prime}(0)-\int_{0}^{t} d s \frac{1}{\sqrt{4 \pi i(t-s)}} \dot{q}(s) .
$$

We apply the operator $I$, defined in (12), and use the charge equation (7) to obtain

$$
\left(I \phi^{\prime}(0, \cdot)\right)(t)=\left(I\left(U(\cdot) \phi_{0}\right)^{\prime}(0)\right)(t)-\frac{1}{\sqrt{4 i}}\left(q(t)-q_{0}\right)=(I \gamma q)(t)
$$

which implies the boundary condition. Here, we used the identities:

$$
I(\pi(\cdot))^{-1 / 2}(t)=\frac{1}{\sqrt{\pi}} \int_{0}^{t} d s \frac{1}{\sqrt{t-s}} \frac{1}{\sqrt{\pi s}}=1 \quad \text { and } \quad I^{2} f(t)=\int_{0}^{t} d s f(s) .
$$

By Eq. (3), to prove the continuity of the map $t \mapsto H_{\gamma(t)} \psi(t)$ in $L^{2}(\mathbb{R})$, it is enough to show the continuity of $\left\|\phi^{\prime \prime}(t)\right\|$. As the continuity of $U(t) \phi_{0}$ is obvious, we just need to show that:

$$
\lim _{\delta \rightarrow 0}\|\hat{\tilde{\phi}}(t+\delta)-\hat{\tilde{\phi}}(t)\|_{L^{2}\left(\mathbb{R},\left|k^{4}\right| d k\right)}^{2}=0 .
$$

By Eqs. (14) and (14a), this is reduced to show that:

$$
\lim _{\delta \rightarrow 0} \int_{\mathbb{R}} d k k^{2}\left|\int_{t}^{t+\delta} d s e^{-i k^{2} s} \dot{q}(s)\right|^{2}=0
$$

For the proof of this statement, we refer to the proof of Prop. 3.3 in [23]. 


\section{Acknowledgements}

The authors acknowledge the support of the FIR 2013 project "Condensed Matter in Mathematical Physics", Ministry of University and Research of Italian Republic (code RBFR13WAET).

\section{References}

[1] Gesztesy F. and Holden H. A new class of solvable models in quantum mechanics describing point interactions on the line, J. Phys. A, 1987, 20(15), P. 5157-5177.

[2] Šba P. Some remarks on the $\delta^{\prime}$-interaction in one dimension, Rep. Math. Phys., 1986, 24(1), P. 111-120.

[3] Albeverio S., Gesztesy F., Hoegh-Krohn R., and Holden H. Solvable Models in Quantum Mechanics, $A M S$, 2005. With an appendix of P. Exner.

[4] Sayapova M. R. and Yafaev D. R. The evolution operator for time-dependent potentials of zero radius, Boundary value problems of mathematical physics, vol. 159, Part 12, Work collection, Trudy Mat. Inst. Steklov., 1983, P. 167-174.

[5] Yafaev D. R. Scattering theory for time-dependent zero-range potentials, Annales de l'IHP Physique théorique, 1984, 40(4), P. 343-359.

[6] Correggi M., Dell'Antonio G., Figari R., and Mantile A. Ionization for Three Dimensional Time-Dependent Point Interactions, Comm. Math. Phys., 2005, 257(1), P. 169-192.

[7] Correggi M. and Dell'Antonio G. Decay of a bound state under a time-periodic perturbation: a toy case, $J$. Phys. A, 2005, 38(22), P. 4769-4781.

[8] Carlone R., Correggi M., and Figari R. Two-dimensional time-dependent point interactions, arXiv:1601.02390 [math-ph], 2016, 17 pp.

[9] Hmidi T., Mantile A., and Nier F. Time-dependent Delta-interactions for 1D Schrödinger Hamiltonians, Math. Phys. Anal. Geom., 2009, 13(1), P. 83-103.

[10] Neidhardt H. and Zagrebnov V. A. Linear non-autonomous Cauchy problems and evolution semigroups, $A d v$. Differential Equations, 2009, 14(3/4), P. 289-340.

[11] Adami R., Dell'Antonio G., Figari R., and Teta A. The Cauchy problem for the Schrödinger equation in dimension three with concentrated nonlinearity, Ann. Inst. H. Poincaré Anal. Non Linéaire, 2003, 20(3), P. 477-500.

[12] Adami R., Dell'Antonio G., Figari R., and Teta A. Blow-up solutions for the Schrödinger equation in dimension three with a concentrated nonlinearity, Ann. Inst. H. Poincaré Anal. Non Linéaire, 2004, 21(1), P. 121-137.

[13] Adami R., Noja D., and Ortoleva C. Orbital and asymptotic stability for standing waves of a nonlinear Schrödinger equation with concentrated nonlinearity in dimension three, J. Math. Phys., 2013, 54(1), P. 013501.

[14] Adami R. and Teta A. A Simple Model of Concentrated Nonlinearity, Mathematical Results in Quantum Mechanics (Dittrich J., Exner P., and Tater M., eds.), Operator Theory Advances and Applications, Birkhäuser Basel, January 1999, P. 183-189.

[15] Adami R. and Teta A. A class of nonlinear Schrödinger equations with concentrated nonlinearity, J. Funct. Anal., 2001, 180(1), P. 148-175.

[16] Holmer J. and Liu C., Blow-up for the 1D nonlinear Schrödinger equation with point nonlinearity I: Basic theory, arXiv:1510.03491 [math.AP], 2015, 22 pp.

[17] Dror N. and Malomed B. A. Solitons supported by localized nonlinearities in periodic media, Phys. Rev. A, 2011, 83(3), P. 033828.

[18] Hennig D., Tsironis G., Molina M., and Gabriel H. A nonlinear quasiperiodic Kronig-Penney model, Phys. Lett. A, 1994, 190(3-4), P. 259-263.

[19] Malomed B. A. and Azbel M. Y. Modulational instability of a wave scattered by a nonlinear center, Phys. Rev. B, 1993, 47(16), P. 10402-10406.

[20] Molina M. and Bustamante C. The attractive nonlinear delta-function potential, Am. J. Phys., 2002, 70, P. 67-70.

[21] Cacciapuoti C. On the derivation of the Schrödinger equation with point-like nonlinearity, Nanonsystems: Phys., Chem., Math, 2015, 6, P. 79-94.

[22] Cacciapuoti C., Finco D., Noja D., and Teta A. The NLS Equation in Dimension One with Spatially Concentrated Nonlinearities: the Pointlike Limit, Lett. Math. Phys., 2014, 104(12), P. 1557-1570. 
[23] Cacciapuoti C., Finco D., Noja D., and Teta A. The point-like limit for a NLS equation with concentrated nonlinearity in dimension three, arXiv:1511.06731 [math-ph], 2015, 35 pp.

[24] Kisyński J. Sur les opérateurs de Green des problemes de Cauchy abstraits, Studia Mathematica, 1964, 3(23), P. 285-328.

[25] Kato T. Linear evolution equations of "hyperbolic" type, J. Fac. Sci. Univ. Tokyo Sect. I, 1970, 17(6), P. 241-258

[26] Dell'Antonio G. F., Figari R., and Teta A., A limit evolution problem for time-dependent point interactions, J. Funct. Anal., 1996, 142(1), P. 249-274.

[27] Bahouri H., Chemin J.-Y., and Danchin R. Fourier analysis and nonlinear partial differential equations, vol. 343, Springer Science \& Business Media, 2011.

[28] Duistermaat J. J. and Kolk J. A. Distributions: Theory and Applications, Springer, 2010.

[29] Lions J. L. and Magenes E. Non-homogeneous boundary value problems and applications, vol. 1, Springer, Berlin, 1972.

[30] Strichartz R. S. Multipliers on fractional Sobolev spaces, J. Math. Mech, 1967, 16(9), P. 1031-1060.

[31] Gripenberg G., Londen S. O., and Staffans O., Volterra integral and functional equations, vol. 34, Cambridge University Press, 1990. 